\title{
Surgical Anatomy of the Cervical Carotid Artery for Carotid Endarterectomy
}

\author{
Nakamasa HAYASHI, Emiko HORI, Yuko OHTANI*, Osamu OHTANI*, \\ Naoya KUWAYAMA, and Shunro ENDO
}

\author{
Departments of Neurosurgery and *Anatomy, Toyama Medical and \\ Pharmaceutical University, Toyama
}

\begin{abstract}
Carotid endarterectomy (CEA) is the main treatment for atherosclerotic plaque of the cervical internal carotid artery. The surgical anatomy of the carotid arteries was studied in the carotid triangle of 49 cadavers. The carotid bifurcation was located at the level of the lower third of C-3. The superior thyroid artery arose from the anterior wall of the external carotid artery in $70 \%$ of specimens and from the distal portion of the common carotid artery in $30 \%$. The lingual artery arose as a separate trunk between the origins of the superior thyroid and facial arteries in $81 \%$ of specimens, with the facial artery from a common trunk in $18 \%$, and with the superior thyroid artery in $1 \%$. The occipital artery arose from the posterior aspect of the external carotid artery above the level of origin of the facial artery in $57 \%$ of specimens, between the origins of the facial and lingual arteries in $32 \%$, and below the origin of the lingual artery in $11 \%$. The origin of the occipital artery was positioned low and the distal portion of the occipital artery was crossed by the hypoglossal nerve in $20 \%$. The ascending pharyngeal artery arose from the posterior wall of the external carotid artery above the level of origin of the lingual artery in $66 \%$ of specimens, below the origin of the lingual artery in $9 \%$, from the proximal portion of the occipital artery in $19 \%$, from the carotid bifurcation in $2 \%$, and from the internal carotid artery in $2 \%$. The branches of the external carotid artery are the key landmarks for adequate exposure and appropriate placement of cross-clamps on the carotid arteries. It is necessary to understand the surgical anatomy of the carotid arteries to carry out successful removal of plaque and minimize postoperative complications in a bloodless surgical field.
\end{abstract}

Key words: anatomy, carotid artery, carotid endarterectomy, surgical technique

\section{Introduction}

Carotid endarterectomy (CEA) is the main treatment for atherosclerotic plaque of the cervical internal carotid artery, which is an important cause of transient ischemic attacks and stroke. Randomized trials have shown that symptomatic patients with at least $70 \%$ stenosis of the internal carotid artery benefit from CEA, and that patients with $50 \%$ to $69 \%$ stenosis and asymptomatic patients experience lesser benefit. $1,5,6,11,13)$ Since the benefit from CEA depends on the incidence of perioperative stroke and death, surgical skill with a low complication rate is absolutely essential. The American Heart Association has published fixed guidelines for CEA. ${ }^{2,12)}$ The surgeon should perform the operation with a rate of perioperative stroke or death of less

Received January 7, 2004; Accepted October 24, 2004 than $6 \%$ for symptomatic patients with severe and moderate carotid stenosis, and $3 \%$ for asymptomatic patients with at least $60 \%$ carotid stenosis.

Vascular control prior to arteriotomy is essential to minimize the ischemic and embolic complications in the CEA. Rapid and accurate removal of atherosclerotic plaque of the internal carotid artery can be only achieved with a bloodless surgical field obtained by cross-clamping of the internal, external, and common carotid arteries. The cross-clamp must be placed beyond the distal extent of the plaque of the internal carotid artery and proximal to any branches of the external carotid artery, to prevent unacceptable backbleeding during the arteriotomy and repair. Therefore, it is important to understand the branching patterns of the branches of the carotid arteries for appropriate dissection of the carotid arteries and placement of the cross-clamp.

The present study examined the surgical anatomy of the carotid arteries located in the carotid triangle 
important for CEA in formalin-fixed specimens.

\section{Methods and Materials}

Ninety-eight carotid arteries were dissected in 49 Japanese cadavers, 25 males and 24 females (mean age 81 years). The bilateral common, external, and internal carotid arteries were dissected. The cervical level of the carotid bifurcation was determined by comparison with the cervical vertebrae. Formalin fixation causes increases in size (2-3\%) of atherosclerotic arteries ${ }^{3)}$ but these increases were negligible in our measurements. The difference in the level of bifurcation between the surgical and angiographic views was examined after arteriotomy of the common and internal carotid arteries in 30 of the 98 specimens. Every branch originating from the carotid arteries located in the carotid triangle was dissected. Branching patterns of the branches of the carotid arteries were examined. The relationship between the origins of the occipital artery and the hypoglossal nerve was also examined.

\section{Results}

\section{Cervical level of the carotid bifurcation}

This study found no difference in level between the two sides (Fig. 1). The carotid bifurcation occurred most frequently at the middle third of C-3, and the mean position of the carotid bifurcation was located at the lower third of C-3. The mean distance between the surgical and angiographic positions of the bifurcation was $3.9 \mathrm{~mm}$ (range 1.0-6.6 $\mathrm{mm}$ ).

II. Branching patterns of the superior thyroid, lingual, and facial arteries

The branches arose as a separate trunk from the external carotid artery in $81 \%$ of specimens (Fig. 2).
The facial artery originated with the lingual artery as a common trunk in $18 \%$, and the lingual artery originated with the superior thyroid artery in $1 \%$. The superior thyroid artery arose from the anterior wall of the external carotid artery in $70 \%$ of our specimens, but arose from the common carotid artery in $30 \%$.

\section{Branching patterns of the occipital artery}

The occipital artery arose above the facial artery in $57 \%$ of specimens, between the facial and lingual arteries in $32 \%$, and proximal to the lingual artery in $11 \%$ (Fig. 3). Usually, the occipital artery is crossed superficially by the hypoglossal nerve at its origin. The origin of the occipital artery was positioned low

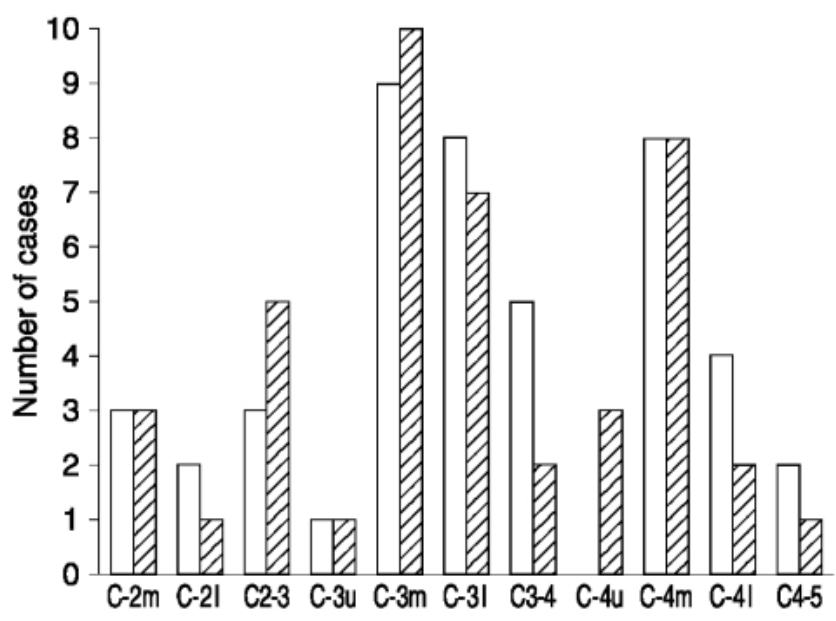

Fig. 1 Distribution of the carotid bifurcation at the cervical level as determined by comparison with the cervical vertebrae. The white column indicates bifurcations on the right side, and the shaded column those on the left side. l: lower, m: middle, u: upper.
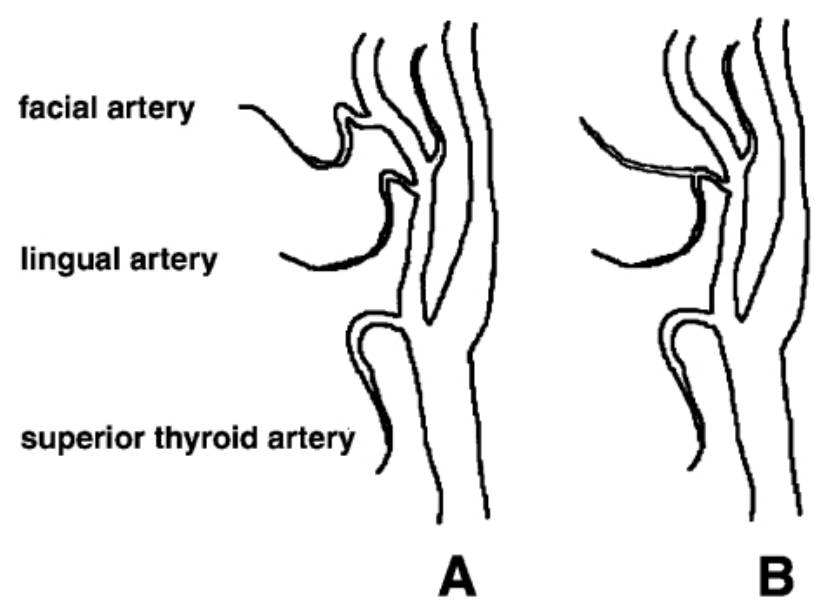
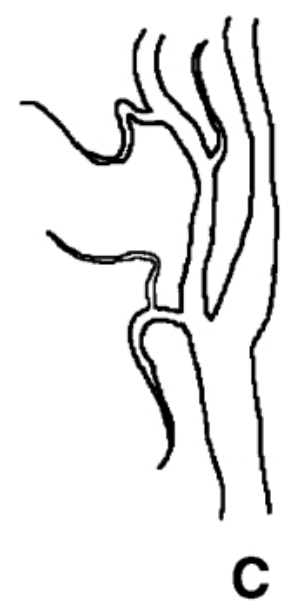

Fig. 2 Schematic drawings of the branching patterns of the superior thyroid, lingual, and facial arteries. A: The branches arise as a separate trunk from the external carotid artery (81\%), B: the facial artery originates with the lingual artery as a common trunk (18\%), and C: the lingual artery originates with the superior thyroid artery (1\%). 


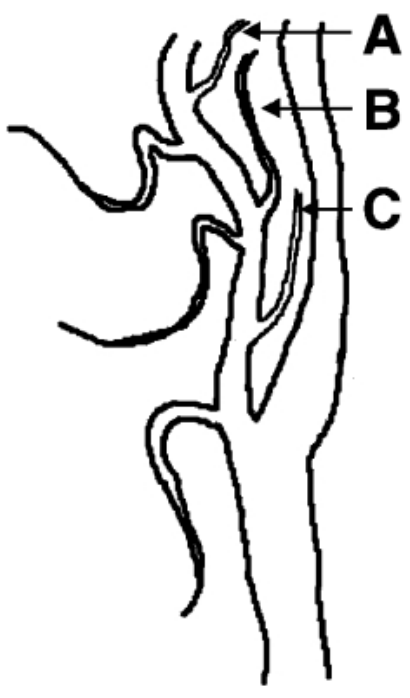

Fig. 3 Schematic drawing of the branching patterns of the occipital artery. The occipital artery arises above the facial artery (A: $57 \%$ ), between the facial and lingual arteries (B: 32\%), and proximal to the lingual artery (C: 11\%).

and the distal portion of the occipital artery was crossed by the hypoglossal nerve in $20 \%$ of specimens.

\section{Branching patterns of the ascending pharyn- geal artery}

The ascending pharyngeal artery usually arose from the posterior wall of the external carotid artery, distal to the lingual artery in $66 \%$ of specimens and proximal to the lingual artery in $9 \%$. The ascending pharyngeal artery originated from the proximal portion of the occipital artery in $19 \%$ of specimens, from the carotid bifurcation in $2 \%$ (Fig. 4A), and from the internal carotid artery in $2 \%$ (Fig. 4B).

\section{Discussion}

The location of the carotid bifurcation and the distal extension of atherosclerotic plaque in the internal carotid artery both contribute to the technical difficulty of CEA. Several methods have been described for added exposure of the distal carotid artery. ${ }^{7,15)}$ If the carotid bifurcation is above C-3 or the plaque extends distally, standard approaches may not provide adequate exposure for removal of the plaque and for arteriotomy repair. ${ }^{7}$ The present study found that the carotid bifurcation of Japanese individuals was most frequently located at the middle third of $\mathrm{C}-3$, and the mean position of the

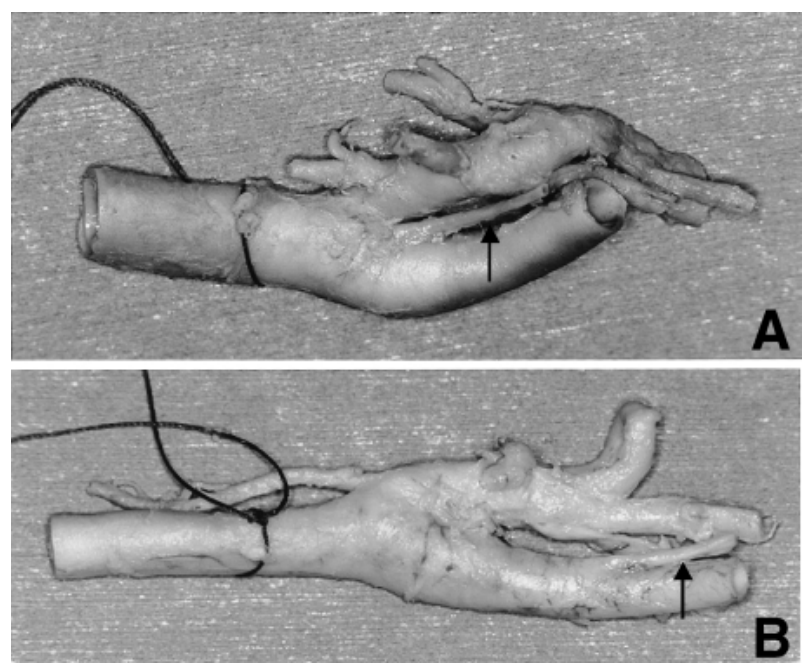

Fig. 4 Photographs showing specimens with the ascending pharyngeal artery originating from the carotid bifurcation (A) and from the internal carotid artery (B).

carotid bifurcation was located at the lower third of C-3. The bifurcation in Europeans is most frequent at the level of the disc between C-3 and C-4. ${ }^{9)}$ The carotid bifurcation is thus located higher in Japanese than in Europeans.

Dissection of the internal carotid artery must be complete and clearly beyond the distal extent of the plaque before cross-clamping and arteriotomy are performed. The position of the carotid bifurcation is determined before surgery from the angiogram. However, the level of bifurcation revealed by preoperative angiography often differs from that recognized at surgery. The difference between the surgical and angiographic views indicated the bifurcation was positioned lower by 1.0 to $6.6 \mathrm{~mm}$ than expected. Angiographically, the carotid bifurcation is usually located at the level of C-4, ${ }^{4}$ whereas, anatomically, the bifurcation is most frequently located at the level of the disc between C-3 and C-4.9)

The distal extent of the plaque is also determined from the angiogram before surgery. The origins of the branches of the external carotid artery act as key landmarks for adequate exposure and appropriate placement of the cross-clamp on the carotid arteries. It is helpful to assess the patterns of the branches of the carotid arteries located in the carotid triangle on the angiogram before surgery. Determination of the distal extent of the plaque in the internal carotid artery by comparison with the position of the origin of the occipital artery should be particularly helpful.

Usually, the occipital artery is crossed superficial- 
ly by the hypoglossal nerve at its origin. The connective tissue surrounding the hypoglossal nerve is dissected and moved upward to adequately expose the internal carotid artery, if high exposure is needed during CEA. If the origin of the occipital artery is low and the distal portion of the occipital artery is crossed by the hypoglossal nerve, sacrifice of the occipital artery and sternocleidomastoid branches may be required before the hypoglossal nerve is moved upward, to prevent paralysis of the hypoglossal nerve.

The ascending pharyngeal artery is frequently said to originate from the back of the external carotid artery near the bifurcation of the common carotid artery. However, the origin and configuration are quite variable, and the origin may be the internal carotid artery. ${ }^{8,14)}$ The meticulous anatomical dissection and identification of vital structures needed to minimize postoperative complications can only be achieved with a bloodless field. ${ }^{10)}$ If the ascending pharyngeal artery arises close to the origin of the external carotid artery proximal to the lingual artery, carotid bifurcation, and internal carotid artery, this vessel must be identified to ensure that no troublesome backbleeding occurs during plaque removal. The ascending pharyngeal artery arose from the carotid bifurcation in $2 \%$ of our specimens, and from the internal carotid artery in $2 \%$. We usually dissect circumferentially around the carotid arteries located in the carotid triangle and try to find any branches arising from the posterior wall of the carotid arteries near the carotid bifurcation. We have experienced only two cases of embolic complication among about 250 cases of CEA $(0.8 \%)$ performed using this technique, but we are convinced that the carotid arteries must be isolated with the gentlest possible dissection.

The branches of the carotid arteries located in the carotid triangle are key landmarks for the adequate dissection of the carotid arteries and should be identified before cross-clamps are placed and arteriotomy is performed.

\section{References}

1) Barnett HJM, Taylor DW, Eliasziw M, Fox AJ, Ferguson GG, Haynes RB, Rankin RN, Clagett GP, Hachinski VC, Sackett DL, Thorpe KE, Meldrum HE, Spence JD: Benefit of carotid endarterectomy in patients with symptomatic moderate or severe stenosis. North American Symptomatic Carotid Endarterectomy Trial Collaborators. N Engl J Med 339: 1415-1425, 1998

2) Biller J, Feinberg WM, Castaldo JE, Whittemore AD, Harbaugh RE, Dempsey RJ, Caplan LR, Kresowik TF, Matchar DB, Toole JF, Easton JD, Adams HP Jr,
Brass LM, Hobson RW II, Brott TG, Sternau L: Guidelines for carotid endarterectomy: a statement for healthcare professionals from a Special Writing Group of the Stroke Council, American Heart Association. Circulation 97: 501-509, 1998

3) Dalager-Pedersen S, Falk E, Ringgaard S, Kristensen IB, Pedersen EM: Effects of temperature and histopathologic preparation on the size and morphology of atherosclerotic carotid arteries as imaged by MRI. J Magn Reson Imaging 10: 876-885, 1999

4) Dilenge D, Heon M: The internal carotid artery, in Newton TH, Potts DG (eds): Radiology of the Skull and Brain, vol 2, book 2. Saint Louis, CV Mosby, 1974, pp 1202-1245

5) European Carotid Surgery Trialists' Group: Randomized trial of endarterectomy for recently symptomatic carotid stenosis: final results of the MRC European Carotid Surgery Trial (ECST). Lancet 351: 1379-1387, 1998

6) Executive Committee for the Asymptomatic Carotid Atherosclerosis Study: Endarterectomy for asymptomatic carotid artery stenosis. JAMA 273: 1421-1428, 1995

7) Frim DM, Padwa B, Buckley D, Crowell RM, Ogilvy CS: Mandibular subluxation as an adjunct to exposure of the distal internal carotid artery in endarterectomy surgery. Technical note. J Neurosurg 83: 926-928, 1995

8) Grand W, Hopkins LN: The external carotid artery, in: Vasculature of the Brain and Cranial Base. New York, Stuttgart, Thieme, 1999, pp 11-19

9) Gray H: The carotid system of arteries, in Williams PL, Warwick R (eds): Gray's Anatomy, ed 36. Edinburgh, London, Melbourne, New York, Churchill Livingstone, 1980, pp 676-692

10) Loftus CM, Quest DO: Technical issues in carotid surgery 1995. Neurosurgery 36: 629-647, 1995

11) Mayberg MR, Wilson SE, Yatsu F, Weiss DG, Messina L, Hershey LA, Colling C, Eskridge J, Deykin D, Winn HR: Carotid endarterectomy and prevention of cerebral ischemia in symptomatic carotid stenosis. Veterans Affairs Cooperative Studies Program 309 Trialists Group. JAMA 266: 3289-3294, 1991

12) Moore WS, Barnett HJM, Beebe HG, Bernstein EF, Brener BJ, Brott T, Caplan LR, Day A, Goldstone J, Hobson RW II, Kempczinski RF, Matchar DB, Mayberg MR, Nicolaides AN, Norris JW, Ricotta JJ, Robertson JT, Rutherford RB, Thomas D, Toole JF, Trout HH III, Wiebers DO: Guidelines for carotid endarterectomy. A multidisciplinary consensus statement from the Ad Hoc Committee, American Heart Association. Stroke 26: 188-201, 1995

13) North American Symptomatic Carotid Endarterectomy Trial Collaborators: Beneficial effect of carotid endarterectomy in symptomatic patients with highgrade carotid stenosis. N Engl J Med 325: 445-453, 1991

14) Teal JS, Rumbaugh CL, Segall HD, Bergeron RT: Anomalous branches of the internal carotid artery. 
Neuroradiology 106: 567-573, 1973

15) Weiss MR, Smith HP, Patterson AK, Weiss RM: Patient positioning and nasal intubation for carotid endarterectomy. Neurosurgery 19: 256-257, 1986
Address reprint requests to: N. Hayashi, M.D., Department of Neurosurgery, Toyama Medical and Pharmaceutical University, 2630 Sugitani, Toyama 930-0194, Japan.

e-mail: nakamasa@iwa.att.ne.jp

Commentary on this paper appears on the next page. 


\section{Commentary}

Carotid endarterectomy is the only treatment proven in randomized trials to reduce the incidence of stroke and transient ischemic attacks in patients with significant carotid stenosis. In order to provide therapeutic benefit, however, the surgeon must be able to perform the endarterectomy with low morbidity or mortality. As the authors have stated, a thorough knowledge of the anatomy of the carotid triangle and its variants along with meticulous dissection is necessary to achieve acceptable results.

In examining the carotid arteries in 49 Japanese cadavers, the authors have elegantly demonstrated the normal anatomy of the cervical triangle as well as the variations in that anatomy that can lead to intraoperative misadventures. Through a better understanding of this variable anatomy and its preoperative recognition, surgeons may better balance the need to completely dissect all branches of the carotid artery yet minimize manipulation that may lead to intraoperative embolic complications.

Daniel L. BARROW, M.D. Department of Neurosurgery The Emory Clinic Atlanta, Georgia, U.S.A.

The authors present a detailed description of the relevant vascular anatomy confronting the surgeon performing a carotid endarterectomy. Hayashi et al. conducted a meticulous anatomical study of the arterial branches, both normal and anatomical variants, of the extracranial carotid system, that is, 98 carotid systems in 49 Japanese cadavers. The large number of specimens examined provides reliability regarding the quoted percentages (incidence) of anatomical variants. Bifurcation of the common carotid artery in the Japanese population was similar bilaterally but higher (mid C-3) compared with that in Caucasians (level of the disc between C-3 and C-4). The bifurcation was also demonstrated angiographically to range from 1 to $6.6 \mathrm{~mm}$ (mean 3.9), lower than the level identified at anatomical dissections. However, the authors neither explain this difference nor its consistency; such an explanation would be valuable.

The authors emphasize the importance of the angiographic identification of all the arterial branches of the extracranial carotid system for preoperative planning. Knowledge of the origin and course of these branches (i.e., ascending pharyngeal, superior thyroid, lingual, facial, and occipital arteries) serve as landmarks during the surgical exposure and crossclamping placement.

The anatomical diligence of this study is laudable. The authors have emphasized the import of a detailed knowledge about the vascular anatomy in carotid endarterectomies. Their demonstration of a difference in level of the carotid artery bifurcation in the Japanese population warrants consideration of this issue in other ethnic populations.

John M. TEW, Jr., M.D., Jeffrey T. KELLER, Ph.D., and Federica BeretTA, M.D.* The Neuroscience Institute: University of Cincinnati College of Medicine and the Mayfield Clinic Cincinnati, Ohio, U.S.A.;

${ }^{*}$ University of Cincinnati College of Medicine Cincinnati, Ohio, U.S.A.

Understanding the surgical anatomy involving the region of carotid bifurcation is the key to success for carotid endarterectomy. The important structures in this region include the superior thyroid artery, the lingual artery, the facial artery, the occipital artery, the ascending pharyngeal artery, and the internal and external carotid arteries as well as the hypoglossal nerve. To identify the variations of the relationships between these structures on the preoperative imaging study and during the operation is important for the decision of the extent of exposure and the site of cross clamping of the arteries.

In the present article, the authors studied the anatomy of carotid bifurcation on 49 cadavers and analyzed the variations of these structures. The authors emphasized that preoperative comparison of the relation between the distal extent of the plaque and the origin of occipital artery is important for adequate surgical exposure. Another important message delivered in this article is that the origin of the ascending pharyngeal artery is very variable. Identification of this artery is important for good hemostasis during the removal of the plaque.

Yong-Kwang TU, M.D. Department of Neurosurgery College of Medicine and Hospital National Taiwan University Taipei, Taiwan, R.O.C. 